\title{
Generalized Hydrodynamics for integrable systems
}

1. Generalized hydrodynamics in strongly interacting 1D Bose gases

Authors: Neel Malvania, Yicheng Zhang, Yuan Le, Jerome Dubail, Marcos Rigol,

David S. Weiss

arXiv:2009.06651

2. Generalized HydroDynamics on an Atom Chip

Authors: Max Schemmer, Isabelle Bouchoule, Benjamin Doyon, Jerome Dubail

arXiv: 1810.07170

and Phys. Rev. Lett. 122, 090601 (2019)

\section{Recommended with a Commentary by Thierry Giamarchi, University of Geneva}

Determining the physical properties of strongly correlated systems is clearly one of the final frontiers of condensed matter and cold atomic gases. For equilibrium systems, although the situation remains complicated in "high" dimensions (in particular in two dimensions), one-dimensional quantum systems have been blessed by various efficient methods: i) field theory with techniques such as bosonization [1] have allowed to get asymptotically exact descriptions of interacting bosonic and fermionic systems; ii) exactly solvable models such as the Lieb-Lininger model [2] for Bosons and the Hubbard model for fermions [3] have allowed to obtain thermodynamic properties and more recently even correlation functions. The have also provided a rock solid basis on which to benchmark the more approximate methods such as field theory; iii) remarkable numerical techniques such as DMRG [4] and its variants [5] have been developed giving access to correlations for reasonable sizes and times both in equilibrium and for out of equilibrium systems. More importantly we have learned to combine these methods, extracting exact parameters to inject in the field theory from the numerics or the Bethe-ansatz [6], turning the field theory into an essentially exact description with spectacular experimental success.

However this fairy tale turns rapidly sour when we move away from equilibrium situations to deal with out of equilibrium ones. One example is the iconic "Quantum Newton craddle" experiment done in the group of D. Weiss [7] where a gaz of interacting bosons is split in two blobs in a parabolic trap. The two blobs are hurled at each other and despite the many interactions between the particles when the blobs overlap, many "undamped" oscillations of the blobs are observed. For experiments similar to those our three pillars of knowledge become rapidly much less efficient. 
- For field theory the initial distribution is quite far from the equilibrium one and certainly involves highly excited states. So field theory is not particularly reliable for these very out of equilibrium situations, even if it can and had been used with great success for more "moderate" out of equilibrium cases.

- Numerics is of course perfectly adapted to deal with such situations, but is in great difficulty in continuum problems and more importantly has difficulties with reaching long time limits. So questions on whether such an out of equilibrium system would ultimately thermalize, go through intermediate but long lived states etc. are very difficult to answer.

- Integrable models per se are a little bit out of their depth there. First even in equilibrium, correlations could only be computed very recently and only for the Lieb-Lininger model and the XXZ chain. Second and more importantly the situations studied more or less always contain elements that make the system non-integrable in a strict sense (such as the parabolic potential in [7]).

Thus to address such out of equilibrium issues one tries to establish a hydrodynamic description of the problem. This is usually what we do for weakly interacting systems, or systems we believe thermalize rapidly locally [8]. We describe the system via a small number of quantities such as the local density, the local velocity and the local energy density (which represent coarse grained quantities on a "large" cell in the sense that it contains many particles but still small compared to macroscopic sizes). If one assumes that each cell thermalizes then this is the standard hydrodynamics description. Then the resulting equations are manageable and we can extract the dynamics of the problem.

For an integrable system this description fails because of the very large number of conserved quantities that are inherent to such systems. A very smart way to solve this problem is to consider that for an integrable system the rapidities that appear in the exact Betheansatz equations play a role similar to momenta for non-interacting systems, something we normally use in Boltzmann like descriptions [9]. It is thus tempting, even if highly non trivial, to build a hydrodynamic equation for the full distribution of the rapidities. This leads to generalized hydrodynamics equations (GHD) [10,11]. This GHD works where the conventional hydrodynamics would simply fail. Indeed GHD properly takes into account the specificities of near integrable systems and the fact that some excitations live essentially forever. It also provides a correct description of all the excitations irrespectively of how high is their energy, something that would be out of reach of a simple field theory such as a Tomonaga-Luttinger liquid description.

The two papers mentioned in this commentary provide experimental setups with 1D bose gases to test for the validity of such a description. I will not go in the details of cold atomics gases [12] and will just mention that the systems realized are near ideal experimental implementations of the Lieb-Lininger model of free bosons interacting with a contact repulsion. The out of equilibrium aspect of the problem is provided by a proper modification of the the trapping potential: increasing the trap strength by a factor of 100 for paper 1) [see e.g. Fig. 1 of paper 1)] and going from a single minimum to a double well minimum for paper 2) [see e.g. Fig. 4 of paper 2)]. These papers then compare the experimental results with the predictions of GHD, either by looking at the time evolution of the density [Fig. 4 of paper 
2)] or even by observing directly the rapidity distribution (using a trick in the expansion of the gas) in paper 1)). A summary of the experimental results and a comparison with theory is given in Fig. 1. The agreement between experiments and the theory is excellent (contrary to what would be the case with simple hydrodynamics equations) and show the power of this mixed method to tackle out of equilibrium physics for systems close to integrability.

There is no doubt that this method will know several developments and that the story is just at the beginning. Quantization has started to enter into play to go beyond the "classical" hydrodynamics version [13]. In a way the GHD provides for out of equilibrium systems the analogue of coupling the Bethe-ansatz equations with the field theory description in the equilibrium case, and similar concepts and synergies between facets of a problem will probably play crucial roles in other systems and perhaps dimensions as well.

\section{References}

[1] T. Giamarchi, Quantum Physics in One Dimension, Vol. 121 of International Series of Monographs on Physics (Oxford University Press, Oxford, 2004).

[2] E. H. Lieb and W. Liniger, Physical Review 130, 1605 (1963).

[3] F. H. L. Essler et al., The One-Dimensional Hubbard Model (Cambridge University Press, Cambridge, 2005).

[4] S. R. White, Physical Review B 48, 10345 (1993).

[5] U. Schollwöck, Annals of Physics 326, 96 (2011).

[6] F. D. M. Haldane, Physical Review Letters 47, 1840 (1981).

[7] T. Kinoshita, T. Wenger, and D. S. Weiss, Nature 440, 900 (2006).

[8] V. N. Popov, Theoretical and Mathematical Physics 11, 565 (1972).

[9] J. M. Ziman, Principles of the Theory of Solids (Cambridge University Press, Cambridge, 1972).

[10] O. A. Castro-Alvaredo, B. Doyon, and T. Yoshimura, Phys. Rev. X 6, 041065 (2016).

[11] B. Bertini, M. Collura, J. De Nardis, and M. Fagotti, Phys. Rev. Lett. 117, 207201 (2016).

[12] I. Bloch, J. Dalibard, and W. Zwerger, Reviews of Modern Physics 80, 885 (2008).

[13] P. Ruggiero, P. Calabrese, B. Doyon, and J. Dubail, Phys. Rev. Lett. 124, 140603 (2020). 

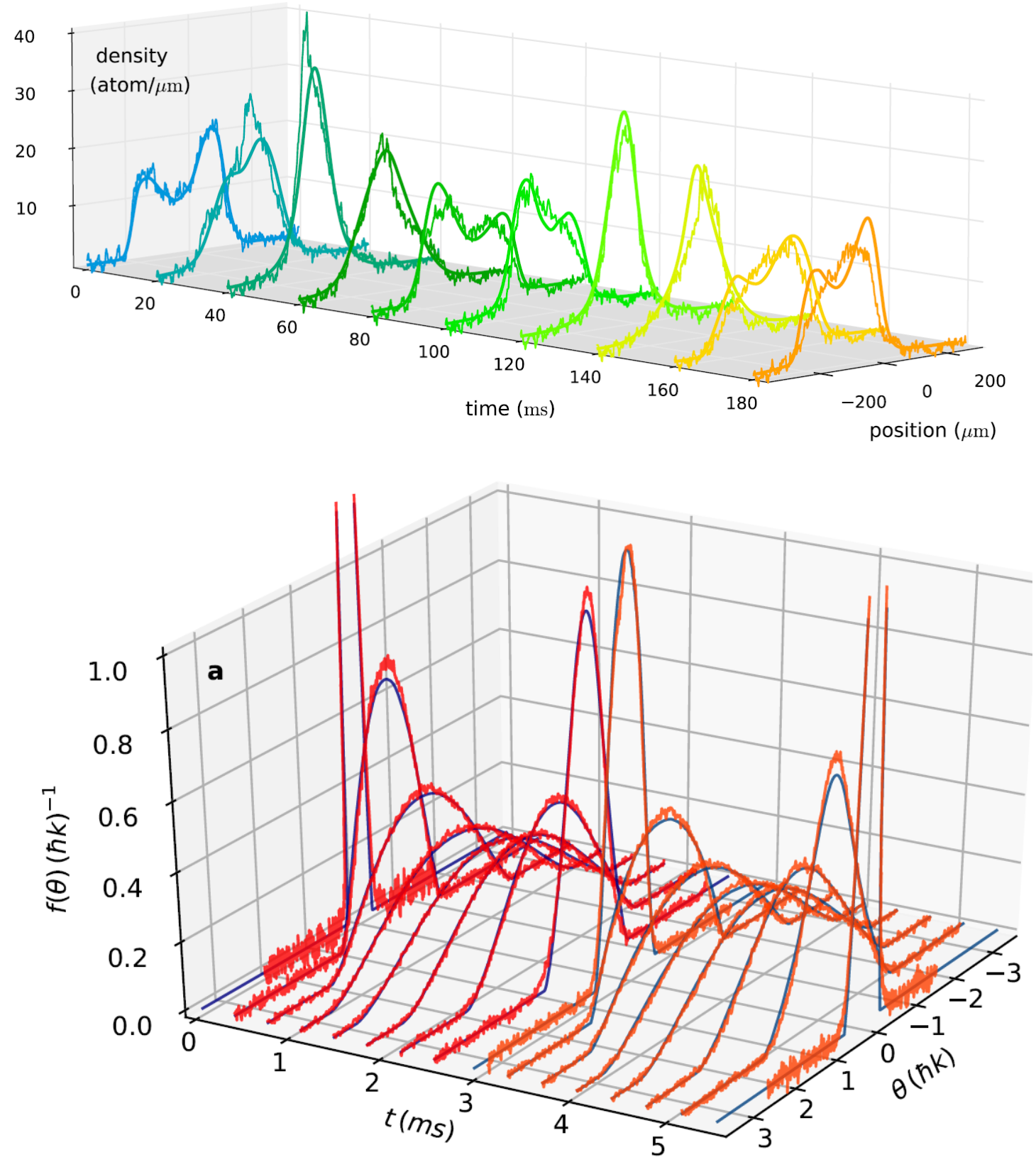

Figure 1: Top [from Fig. 4 of paper 2)]: the evolution of the density of atoms after a quench from a double well to a single well harmonic trap. The solid lines are the predictions of GHD. Bottom [from Fig. 3 of paper 1)]: Evolution of the rapidity distribution after a quench where the depth of the trap has been increased a 100 times. The orange and red curves are the experimental data, the blue curves are the prediction of the GHD theory. In both cases the agreement is clearly remarkable. 